\title{
Time-division multiplexing operation of temperature-compensated fiber Bragg grating underwater acoustic sensor array with feedback control
}

\author{
Hiroki Yokosuka*, Satoshi Tanaka and Nobuaki Takahashi \\ Department of Communications Engineering, National Defense Academy, \\ 1-10-20, Hashirimizu, Yokosuka, 239-8686 Japan
}

(Received 21 February 2005, Accepted for publication 21 March 2005)

Keywords: Underwater acoustic sensor, Optical fiber sensor, Fiber Bragg grating,

Time-division multiplexing, Temperature compensation PACS number: 43.38.Zp, 43.30.Xm [DOI: 10.1250/ast.26.456]

\section{Introduction}

An optical underwater acoustic sensor with a fiber Bragg grating (FBG) as a sensing element is attractive because of features such as a capability of remote sensing and immunity to electromagnetic interference [1-4]. In our laboratory, we have developed the FBG underwater acoustic sensors based on the intensity-modulation scheme [4-6]. Although it could successfully measure the amplitude and phase of sound in water in real time, it is desirable, for wide-area marine sound monitoring, to develop a sensor that can detect sound at more than one location. Therefore, we propose and demonstrate an FBG underwater acoustic sensor array that detects the acoustic field at multiple points by the time-division multiplexing (TDM) technique. Furthermore, to compensate the temperature dependence of an FBG, which may cause the sensor sensitivity to be unstable, a feedback circuit is utilized to control the optical source wavelength for stable operation of the sensor under various temperature environments.

\section{Principle}

The principle of sound detection is based on the intensity modulation scheme [4]. Namely, narrow-band light, such as laser light, the wavelength of which is tuned to the slope of the reflectance spectrum curve of an FBG, is incident on the FBG under the influence of sound pressure and is intensity modulated when it is reflected from the FBG. The intensity modulation is synchronized with the applied sound pressure, and direct photodiode detection of the reflected light allows us to observe the amplitude and phase of the acoustic field in real time. Because the sensor sensitivity depends on temperature, a feedback circuit is attached to the optical source to stabilize the sensitivity. The principle of this sensitivity stabilization is essentially the same as that used for the thermally stabilized FBG vibration sensor [7] but the servomotor system is replaced by an electrical circuit to improve the performance. TDM operation of the sensor is realized here by inserting an optical switch between the optical source and each FBG. By flipping the switch into each channel to which an FBG is connected, the respective FBG is interrogated with the incident light. Since all the FBGs are interrogated with one laser light source, their reflectance spectra must have over-

*e-mail: yokosuka@nda.ac.jp lapping wavelengths. Moreover it is preferable, but not necessary, that all the FBGs have the same reflectance at the operation wavelength. This is because the sensitivity stabilization system automatically controls the source wavelength so that the dc component of the detected sensor output is fixed at a predetermined value. If there is a difference in reflectance between the FBGs, the stabilization system shifts the source wavelength each time the switch is flipped.

\section{Experiment}

Figure 1 shows the experimental setup for the TDM FBG underwater acoustic sensor array. A distributed feedback (DFB) laser diode is used as an optical source that can emit light of up to several milliwatts. The wavelength of the laser can be adjusted from 1,555 to $1,560 \mathrm{~nm}$ by changing the element temperature from 0 to $50^{\circ} \mathrm{C}$. The incident light can be directed arbitrarily to either $\mathrm{FBG}_{1}$ in Sensor1 or $\mathrm{FBG}_{2}$ in Sensor2 by applying the control voltage to the switch. The light reflected from the FBGs is detected by a photodiode to yield the sensor output as well as feedback control signal. Since the feedback circuit involves a low pass filter with the cut-off frequency of $14 \mathrm{~Hz}$, the variation in the photodiode current faster than several hertz is eliminates in the circuit, i.e., the sound component of the current is not allowed to come into the circuit. The reflectance spectra of the FBGs at $28^{\circ} \mathrm{C}$ are shown in Fig. 2. The Bragg wavelengths are $1,556.80 \mathrm{~nm}$ and $1,556.85 \mathrm{~nm}$ for $\mathrm{FBG}_{1}$ and $\mathrm{FBG}_{2}$, respectively. The bandwidths of $\mathrm{FBG}_{1}$ and $\mathrm{FBG}_{2}$ are $1.30 \mathrm{~nm}$ and $1.31 \mathrm{~nm}$, respectively. The pressure and temperature sensitivities of a typical FBG have been measured in a separate experiment. They are $-6 \mathrm{pm} / \mathrm{MPa}$ and $15 \mathrm{pm} /$ degree, respectively [8]. As seen in the figure, the two spectra are almost completely overlapped with little discrepancy. The spectrum of the optical source, the wavelength of which is tuned to the slope of the two spectra so as to satisfy the operation condition of the sensor is also shown. The two FBGs are each placed into a water vessel filled with water and subjected to sound pressure produced by PZT transducers placed at the bottom of the vessels. An optical coupler and optical spectrum analyzer (OSA) are included to monitor the spectrum of the light. The waveform and magnitude of the sensor output are observed on an oscilloscope and electrical spectrum analyzer, respectively. 


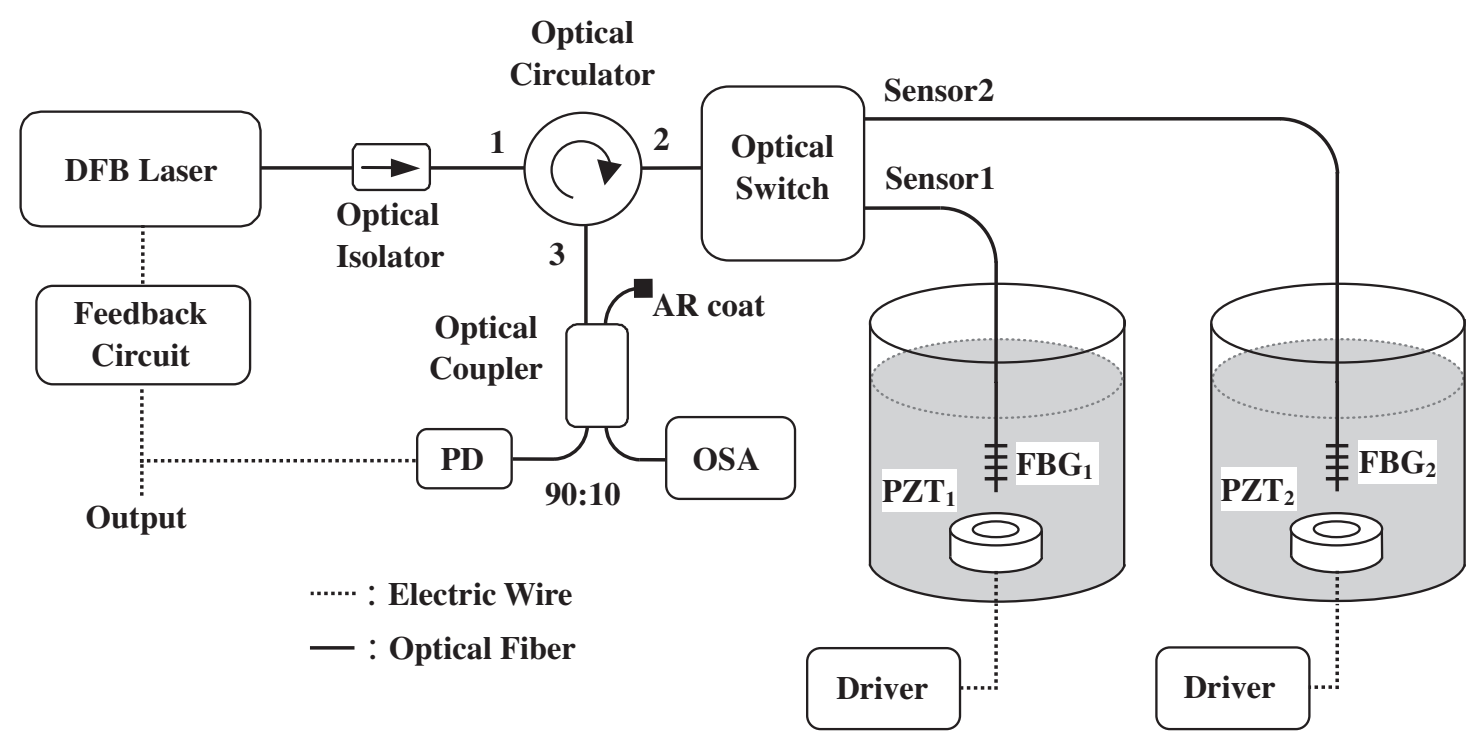

Fig. 1 Experimental setup of TDM FBG underwater acoustic sensor array.

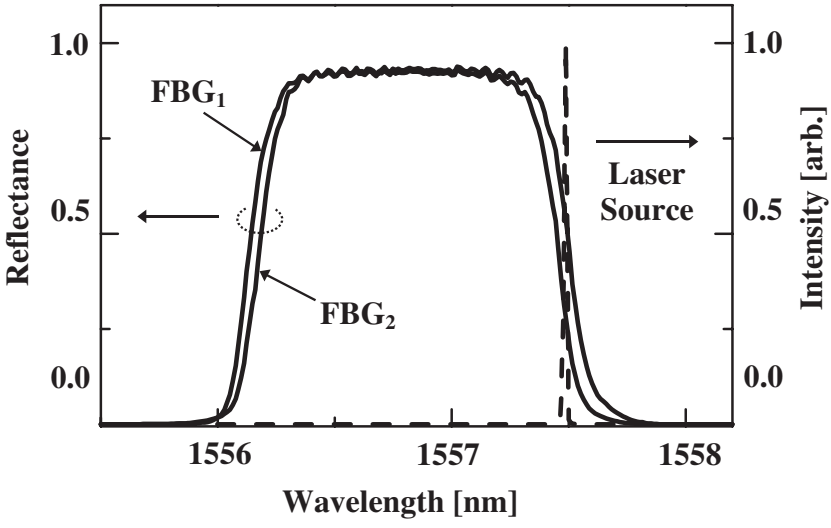

Fig. 2 Reflectance spectra of FBGs and laser source spectrum.

\section{Results and discussions}

Figures 3(a) and 3(b) show the waveforms of the sensor output at room temperature. $\mathrm{PZT}_{1}$ and $\mathrm{PZT}_{2}$ are driven at $16.5 \mathrm{kHz}$ and $16.6 \mathrm{kHz}$, respectively. The outputs from Sensor1 and Sensor2 are observed to be stable in time. The distortion of the waveforms appears to be small, and the sensor is expected to exhibit a linear response. The magnitude of Sensor1 output is plotted as a function of sound pressure in Fig. 4. As expected, the sensor output is proportional to the sound pressure. In order to confirm that the wavelength control feedback circuit is effective in the array system, we observed the sensor output when the direction of the light was switched between Sensor1 and Sensor2 every 25 seconds without applying sound pressure to the FBGs. The result is shown in Fig. 5. In the period of the first 110 seconds, the feedback circuit to control the laser wavelength is disconnected, while the feedback is on the rest of the time. When the feedback is off, the sensor yields two levels of dc output because $\mathrm{FBG}_{1}$ and $\mathrm{FBG}_{2}$ have different reflectance at a fixed wavelength, as was seen in Fig. 2. When the feedback is on, on the other hand, the sensor output is constant except during
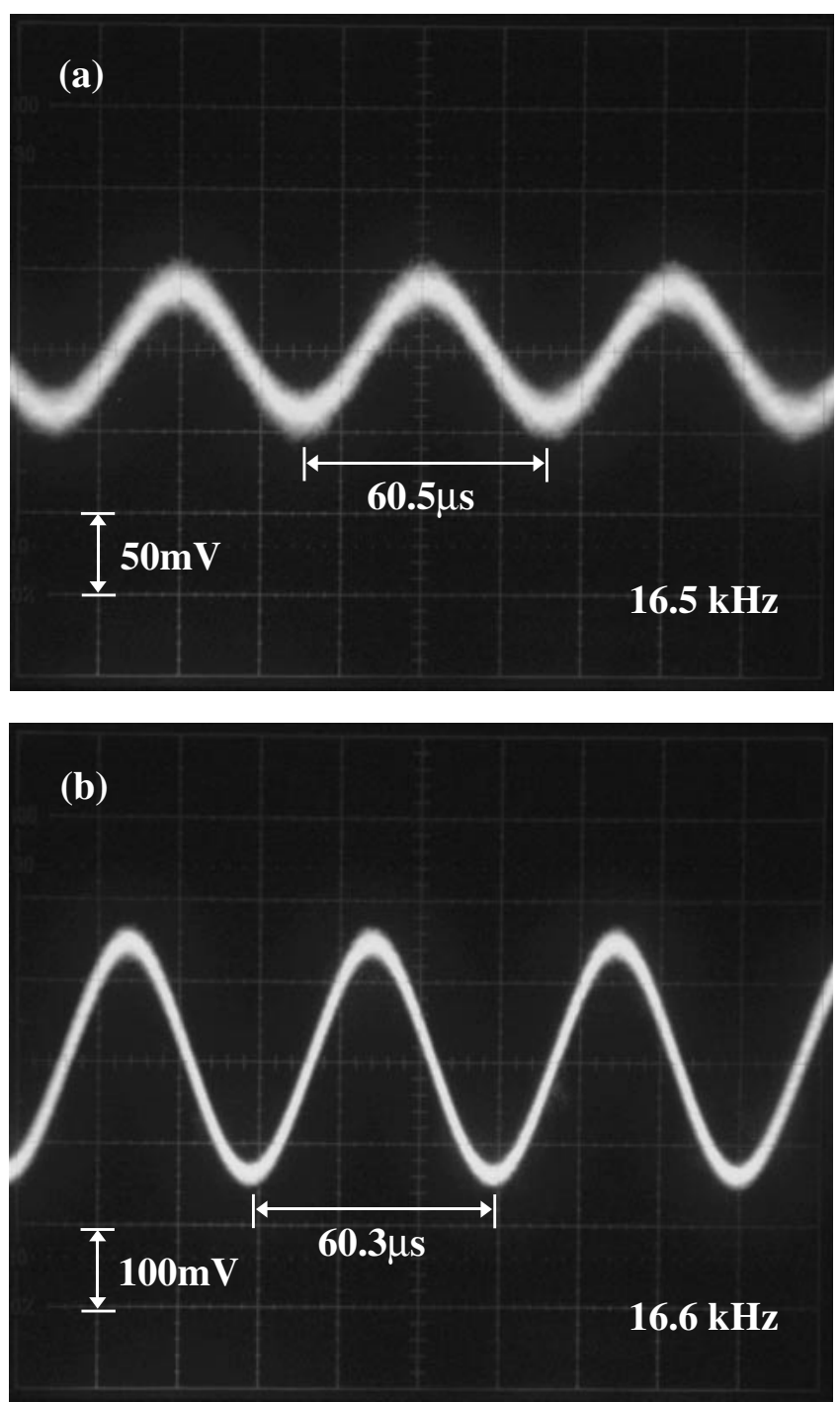

Fig. 3 Waveforms of sensor output: (a) Sensor1 and (b) Sensor2. 


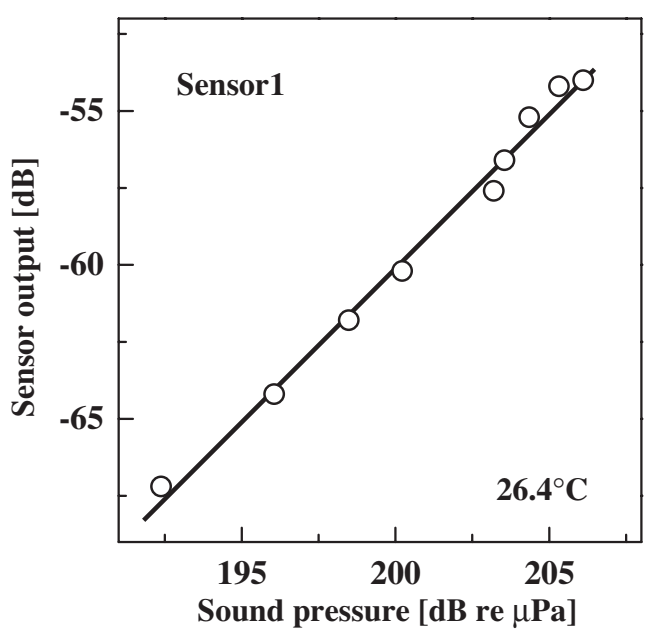

Fig. 4 Sensor output vs sound pressure.

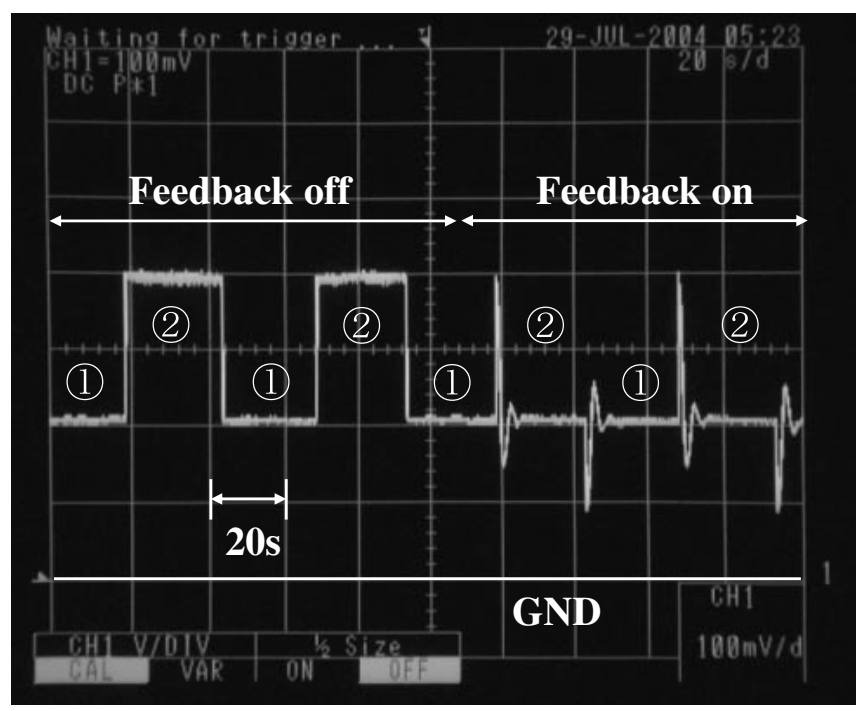

Fig. 5 Sensor output: (1) and (2) correspond to the output from Sensor1 and Sensor2, respectively.

the transient variation caused by switching. This confirms that the feedback circuit automatically controls the laser wavelength until the reflectance of $\mathrm{FBG}_{2}$ becomes the same as that of $\mathrm{FBG}_{1}$ at the laser wavelength. Using two identical FBGs for $\mathrm{FBG}_{1}$ and $\mathrm{FBG}_{2}$ would minimize the spikes in the transient period. With the feedback circuit on and off, we next measured the magnitude of the Sensor1 signal output at $19.6 \mathrm{kHz}$ while varying the temperature around $\mathrm{FBG}_{1}$. The results are shown in Fig. 6. When the feedback control is on, the sensor output is stable against temperature variation and its fluctuation is reduced to $2.5 \mathrm{~dB}$, although it is more than $40 \mathrm{~dB}$ without feedback control.

\section{Summary}

The TDM technique is applied to an FBG underwater acoustic sensor for multipoint monitoring of an acoustic field in water. In order to compensate the temperature dependence

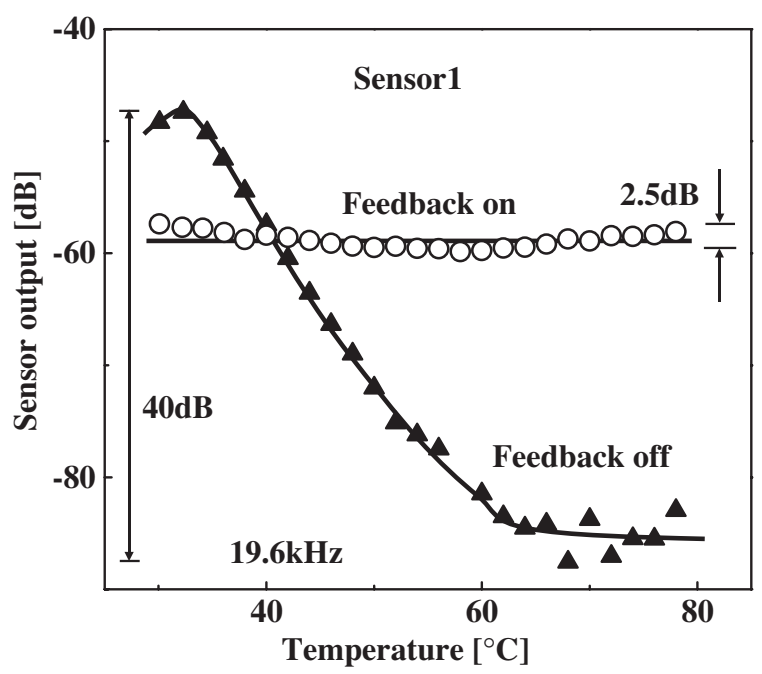

Fig. 6 Temperature dependence of FBG acoustic sensor output.

of the FBG sensing element, the laser source wavelength is automatically controlled by a feedback circuit. The sensor could be used to measure the acoustic field at two points at various temperatures. We obtain a temperature-induced fluctuation of sensor sensitivity of $2.5 \mathrm{~dB}$, although it is more than $40 \mathrm{~dB}$ when the sensor is not equipped with the feedback circuit. Although we demonstrated two-point underwater acoustic sensing using a two-channel optical switch in the present system, it should be easy to use an optical switch for more than two channels to realize acoustic sensing for a large number of points.

\section{References}

[1] P. St. J. Russell and J. L. Archambault, "Fiber Gratings," in Optical Fiber Sensors, Vol. 3, B. Culshaw and J. Dakin, Eds. (Artech House, Boston, 1996), Chap. 2, pp. 9-67.

[2] A. D. Kersey, M. A. Davis, H. J. Patrick, M. LeBlanc, K. P. Koo, C. G. Askins, M. A. Putnam and E. J. Friebele, "Fiber grating sensors," J. Lightwave Technol., 15, 1442-1463 (1997).

[3] N. Takahashi, W. Thongnum and S. Takahashi, "Fiber-Bragggrating vibration sensor with temperature stability using wavelength-variable incoherent light source," Acoust. Sci. \& Tech., 23, 353-355 (2002).

[4] N. Takahashi, A. Hirose and S. Takahashi, "Underwater acoustic sensor with fiber Bragg grating," Opt. Rev., 4, 691694 (1997).

[5] N. Takahashi, K. Yoshimura, S. Takahashi and K. Imamura, "A compact optical fiber hydrophone using fiber Bragg grating," J. Marine Acoust. Soc. Jpn., 27, 28-34 (2000).

[6] N. Takahashi, K. Yoshimura, S. Takahashi and K. Imamura, "Development of an optical fiber hydrophone with fiber Bragg grating," Ultrasonics, 38, 581-585 (2000).

[7] N. Takahashi, W. Thongnum, T. Ogawa, S. Tanaka and S. Takahashi, "Thermally stabilized fiber-Bragg-grating vibration sensor using servo motor control," Opt. Rev., 10, 106-110 (2003).

[8] N. Takahashi, T. Saeki, K. Tetsumura, S. Takahashi and K. Imamura, "Pressure and temperature dependence of fiber Bragg grating for acoustic sensing," J. Marine Acoust. Soc. Jpn., 26, 231-238 (1999). 